$\underline{\text { Fibers and Polymers }}$

July 2017, Volume 18, Issue 7, pp 1255-1262

https://doi.org/10.1007/s12221-017-6862-z

https://link.springer.com/article/10.1007/s12221-017-6862-z

\title{
Characterization of the surface properties of cellulosic fibres in fibrous and ground forms by IGC and contact angle measurements
}

\author{
Erika Fekete $^{1,2^{*}}$ and Emília Csiszár ${ }^{2}$
}

${ }^{1}$ Institute of Materials and Environmental Chemistry, Research Centre for Natural Sciences, Hungarian Academy of Sciences, H-1525 Budapest, P.O. Box 17, Hungary

e-mail: ebodine@mail.bme.hu

${ }^{2}$ Department of Physical Chemistry and Materials Science, Budapest University of Technology and Economics, H-1521 Budapest, P.O. Box 91, Hungary;

e-mail: ecsiszar@mail.bme.hu

*Corresponding author: Phone: +36-1-463-4335, Fax: +36-1-463-3474, address: Department of Physical Chemistry and Materials Science, Budapest University of Technology and Economics, H-1521 Budapest, P.O. Box 91, Hungary, Email: ebodine@mail.bme.hu 
Running head: Surface properties of cellulosic substrates

\begin{abstract}
Surface properties of fibrous and ground cotton and linen were investigated by inverse gas chromatography (IGC) and the contact angle with different liquids was also measured on fabrics of both fibres. Results proved that dispersion component of surface tension $\left(\gamma_{s}{ }^{d}\right)$ determined by IGC depends not only on the surface energy, but also on several factors influencing the adsorbability of probe molecules on the cellulosic substrates. For cotton samples, the trapping of n-alkanes among waxy molecules in the outer layer of fibres can be presumed. This effect results in larger $\gamma_{s}{ }^{d}$ for cotton fibres than for linen in spite of the higher wettability of the linen fabrics. Besides the surface energy and trapping effects, the grinding also influences the $\gamma_{s}{ }^{d}$ values. Specific enthalpy of adsorption $\left(\Delta H_{A}{ }^{a b}\right)$ of polar probes could be determined on all linen samples, but only on the ground cotton sample. Lewis acid-base character calculated for linen and ground cotton samples depends on the same effects as the $\gamma_{s}{ }^{d}$ does. The similar $\Delta H_{A}{ }^{a b}$ values of chloroform (acidic) and THF (basic) measured on each of the samples support the conclusion that the surface character is amphoteric, which is also proved by the high $\Delta H_{A}{ }^{a b}$ values of the amphoteric ethyl acetate and acetone probes.
\end{abstract}

\title{
Keywords:
}

- Inverse gas chromatography

- Dispersion component of surface tension

- Cotton

- Linen

- Contact angle measurement 


\section{INTRODUCTION}

Cellulose based materials such as wood, cotton, linen and hemp have been used for thousands of years in the field of forest products, paper, textiles, etc. Recently, they are widely used for cellulose-reinforced composites with synthetic or biopolymer matrices, because they are biodegradable with low environmental and human health risks [1]. Since their application has broadened continuously, characterization of the surface properties is becoming increasingly important. In the last decades, inverse gas chromatography (IGC) especially at infinite dilution is considered to be a versatile, powerful, sensitive and relatively fast technique for characterization of the physicochemical properties of solid materials. Several papers have been published on surface properties of cellulosic materials and the information collected in this field is increasing significantly [2-19].

Notwithstanding that a series of papers appeared addressing the determination of dispersion component of surface tension and acid-base parameters by IGC, difficulties still exist, since calculation techniques applied for example in determination of the acid-base parameters are not always unambiguous, and sometimes the authors do not define even the units of the reported acidbase parameters. Furthermore, chemical composition, crystallinity and surface morphology of the cellulose based materials are other factors that influence the reported IGC results $[2,16]$. Therefore it is not surprising that somewhat different results for similar materials can be found in the scientific literature.

Recently, we have investigated differently pretreated cotton and linen samples in order to determine and compare the surface energy and acid-base character of the fibres. In the first experiment, fibres from alkaline and enzyme treated cotton fabrics were tested by IGC [20]. The applied treatments i.e. alkaline scouring and bioscouring (i.e. pectinase treatment) are widely used for removing almost totally or partially, respectively, the waxy materials from the outer layer of 
the cotton fibres. Although the measured dispersion component of surface tension $\left(\gamma_{s}{ }^{d}\right)$ was in a good agreement with the experimental results of other measurements and revealed that the alkaline treated cotton is more hydrophilic than the enzyme treated, surprisingly, the difference in $\gamma_{s}{ }^{d}$ as a result of the applied treatments was small $\left(3-4 \mathrm{~mJ} / \mathrm{m}^{2}\right)$. In the light of the fact that the difference in presumable surface energy between the cellulose and the waxy coverage is significant, we expected much higher difference for the alkaline treated and enzyme treated cotton samples. Furthermore, the polar probes were not retained at all on the raw (untreated) cotton, and only a low retention and exclusively at low temperatures could be detected for the treated samples that revealed a basic character.

Assuming that the reason of the weak polar probe retention is the small accessible internal surface of the samples, in the second experiment the IGC measurements was carried out on ground cotton and the study was extended to fibrous and ground linen as well. In spite of the results obtained from numerous classical analytical and high performance methods proved that the new surfaces created by grinding were rich in oxygen, lower values of the dispersion component of surface tension were measured for the ground cotton and linen samples than for the fibres. Furthermore, higher $\gamma_{s}{ }^{d}$ were calculated for cotton than for linen [21]. Based on the X-ray diffraction analysis of fibres and their ground powders it was assumed that the reason of unreal surface energies can be the less effective adsorption of probes on the less perfect crystalline surface of ground samples. Results of the next comprehensive study on ground flax of different size confirmed our statement that the multicellular and multicomponent flax is very susceptible to grinding and undergoes complex chemical and physical changes [22]. All the applied methods revealed differences in properties of the fibrous and ground flax, and the results showed that dispersion components of surface tensions measured by IGC depends on several parameters (not only on surface energy) which influence the adsorption of probe molecules on the surface of 
investigated samples. The grinding has also been reported to affect the surface properties assessed by IGC of other materials [23].

In the light of the results and observations discussed above, an important question is still open: why was so high $\gamma_{s}{ }^{d}$ measured for the raw cotton by IGC? In this paper we report the results on an investigation of this question using cotton and linen in fibrous and ground forms and characterizing the dispersion components of surface tensions and acid-base character by IGC. Additionally, results from contact angle measurements on the fabrics of both fibres also contribute to answer the question.

\section{MATERIALS AND METHODS}

\subsection{Materials}

Raw cotton and linen fibres in fibrous and ground form were used for the IGC test. The fibres were obtained from the respective raw fabrics (cotton: $155 \mathrm{~g} / \mathrm{m}^{2}$, plain weave; linen: 250 $\mathrm{g} / \mathrm{m}^{2}$, plain weave) kindly provided by Pannon-Flax Linen Weaving Co. Győr, Hungary. The fibres were cut into lengths of 1-3 mm and ground in a Mixer Mill MM400 (Retsch GmBH, Germany) at a frequency of $30 \mathrm{1} / \mathrm{s}$, for $2.5 \mathrm{~min}$. The ground fraction studied in this work was composed of particles with diameters enclosed between 200 and $315 \mu \mathrm{m}$, collected by sieving. Swatches of raw fabrics were used in contact angle measurement. The analytical grade chemicals for contact angle measurement and the chromatography grade probes used for IGC were purchased from SigmaAldrich Chemicals. The parameters of solvents used as probes in IGC are listed in Table 1.

\section{Table 1.}

\subsection{Inverse Gas Chromatography}

The fibrous and ground cellulosic fibres (approximately $1.5 \mathrm{~g}$ ) were packed into a stainless steel column having an inner diameter of $5 \mathrm{~mm}$ and a length of $50 \mathrm{~cm}$, and conditioned at $105{ }^{\circ} \mathrm{C}$ 
for $16 \mathrm{~h}$ under a constant flow of nitrogen. For fibrous samples, the packing was performed directly from the yarn by pulling it through the column with the help of a steel wire. IGC measurement was carried out using a Perkin-Elmer Autosystem XL apparatus in the temperature range between 30 and $70{ }^{\circ} \mathrm{C}$ with increments of $10{ }^{\circ} \mathrm{C}$. Vapour samples of 5-20 $\mu \mathrm{l}$ were injected into the column and retention peaks were recorded by a FID detector. High purity nitrogen was used as carrier gas, and its flow rate changed between 5 and $20 \mathrm{~mL} / \mathrm{min}$ depending on measurement temperature and on the type of adsorbent. A new column was prepared for each set of experiments. Each reported value is the result of at least three parallel measurements.

\subsection{Contact angle measurements}

Contact angle measurements were carried out at $23{ }^{\circ} \mathrm{C}$ and $55 \%$ relative humidity using a Ramé-Hart Contact angle goniometer (USA) with a drop image standard software of DT-Acquire and a camera. Liquid drops of $20 \mu 1$ were deposited on each fabric sample and the image of the drops deposited onto the fabric surface was captured immediately by the camera. The value reported is the average of at least 5 readings for each of the samples.

\section{BACKGROUND OF IGC AT INFINITE DILUTION}

The IGC technique using infinite dilutions is a fast, accurate and relatively simple method for the study of the energetics of solid surfaces. According to the principle of the method, the column is packed with the solid to be characterized and probe molecules with known thermodynamic characteristics are made adsorbed on its surface. Surface characteristics can be derived from retention times or volumes. With this technique the dispersion component of the surface tension of the adsorbent can be determined in two ways. Both approaches are based on the fact that the free energy of adsorption $\left(\Delta G_{A}\right)$ is related to the net retention volume $\left(V_{N}\right)$ [24], i.e.

$$
\Delta G_{A}=-R T \ln V_{N}+C
$$


where $C$ is a constant depending on the reference state selected, $R$ is the universal gas constant and $T$ the temperature of the column. The relationship between the free energy $\left(\Delta G_{A}\right)$ and the reversible work of adhesion $\left(W_{A}\right)$ is given by Eq. 2

$$
-\Delta G_{A}=N a_{L V} W_{A}
$$

where $N$ is Avogadro's number and $a_{L V}$ is the molecular surface area of the adsorbate. The free energy and reversible work of adhesion can be divided into two parts: a dispersion term $\left(\Delta G_{A}{ }^{d}\right.$, $\left.W_{A}{ }^{d}\right)$ and the one characterizing the electron donor-acceptor interaction $\left(\Delta G_{A}^{a b}, W_{A}^{a b}\right)$ of the components

$$
\begin{gathered}
W_{A}=W_{A}^{d}+W_{A}^{a b} \\
\Delta G_{A}=\Delta G_{A}^{d}+\Delta G_{A}^{a b}
\end{gathered}
$$

as the $W_{A}{ }^{d}$ is defined by Fowkes approach [25]

$$
W_{A}^{d}=2\left(\gamma_{1}^{d} \gamma_{2}^{d}\right)^{1 / 2}
$$

if we use n-alkanes as probe molecules $W_{A}^{a b}$ equals zero and the dispersion component of the surface tension of the stationary phase can be deduced from the cross-sectional area of n-alkanes and from their surface tension by using Eqs. 1-5. Thus the basic equation of the first approach (Schultz method [26]) to determine $\gamma_{s}^{d}$ takes the following form

$$
R T \ln V_{N}=2 N\left(\gamma_{s}^{d}\right)^{1 / 2} a_{L V}\left(\gamma_{L V}^{d}\right)^{1 / 2}+C
$$

where $\gamma_{s}^{d}$ is the dispersion component of the surface tension of the adsorbent to be determined and $\gamma_{L V}{ }^{d}$ is that of the probe.

The second approach to determine $\gamma_{s}^{d}$ was proposed by Dorris and Gray [27]. If the value of $R T \ln V_{N}$, which is derived from the retention volumes measured with n-alkanes of different chain 
lengths, is plotted against the number of carbon atoms in their chain, we obtain a straight line, the slope of which is given by Eq. 7

$$
-R T \ln \frac{V_{N}^{n}}{V_{N}^{n+1}}=2 N\left(\gamma_{s}^{d}\right)^{1 / 2} a_{C_{2}}\left(\gamma_{C_{2}}\right)^{1 / 2}
$$

where $V_{N}^{n}$ and $V_{N}^{n+1}$ are the retention volumes of n-alkanes with $n$ and $n+1$ carbon atoms, respectively, $a_{\mathrm{CH}_{2}}$ is the surface area occupied by a $-\mathrm{CH}_{2}-$ group and $\gamma_{\mathrm{CH}_{2}}$ is the surface tension of polyethylene.

\section{Acid-base interaction $\left(\Delta G_{A}{ }^{a b}, \Delta H_{A}{ }^{a b}\right)$}

If the adsorbate also enters into interaction with the adsorbent through other than dispersion forces, $\Delta G_{A}{ }^{a b}$ becomes different from zero and the component associated with acid-base interactions is related to the retention volume of the polar probe in the following way

$$
\Delta G_{A}^{a b}=-\left[\left(R T \ln V_{N}+C\right)-\left(R T \ln V_{N}^{r e f}+C\right)\right]=-R T \ln \frac{V_{N}}{V_{N}^{r e f}}
$$

where $V_{N}$ is the net retention volume measured with the probe, while $V_{N}^{r e f}$ is the retention volume, which we would measure if the solvent entered only into dispersion interaction with the adsorbent. $V_{N}^{r e f}$ can be determined from any physico-chemical characteristics of solvents, which is closely related to their dispersion interaction potential, i.e. to their willingness to enter into dispersion interaction with the solid surface $[28,29] . \Delta H_{A}^{a b}$ can be calculated from free energy values determined at different temperatures, since the two quantities are related to each other by Eq. 9 .

$$
\Delta G_{A}^{a b}=\Delta H_{A}^{a b}-T \Delta S_{A}^{a b}
$$

where $\Delta S_{A}{ }^{a b}$ is the entropy of the acid-base interaction of the components. Plotting $\Delta G_{A}{ }^{a b} / T$ against $1 / T$ yields a straight line with the slope of $\Delta H_{A}{ }^{a b}$. 


\section{Donor and acceptor numbers}

The enthalpy of acid-base interaction $\left(\Delta H_{A}{ }^{a b}\right)$ can be also derived from the acid-base parameters of the components according to the theories of Drago [30] or Gutmann [31], respectively. The donor-acceptor approach frequently used for the characterization of acid-base interactions was first suggested by Gutmann [31]. He described all compounds by an acceptor $(A N)$ and a donor $(D N)$ number, which indicate the Lewis acid or base character of a given component. According to his theory, $\Delta H_{A}{ }^{a b}$ is defined in the following way

$$
\Delta H_{A}^{a b}=\frac{(A N)_{A}(D N)_{B}}{100}
$$

He used different methods for the determination of $D N$ and $A N$. His donor numbers are measured by calorimetry and given in $\mathrm{kcal} / \mathrm{mol}$ units. On the other hand, $A N$ is a dimensionless quantity, which is measured by ${ }^{31} \mathrm{P}$ NMR spectroscopy. The value of 100 in the denominator of Eq. 10 accounts for the difference caused by the dissimilar techniques used for the determination of $A N$ and $D N$. Because of the complications caused by the different techniques of determination and the resulting dissimilar dimensions of the $A N$ and $D N$ values obtained, often modified parameters are proposed and used in practice [28]. Riddle and Fowkes [32] proposed a new AN value, which is expressed in $\mathrm{kcal} / \mathrm{mol}$ units and denoted as $A N^{*}$.

Based on Gutmann's theory [31], the most frequently used formula for the determination of the acid-base constants of polymers or inorganic materials is

$$
\Delta H_{A}^{a b}=K_{A} D N+K_{D} A N^{*}
$$

where $K_{A}$ and $K_{D}$ are constants characterizing the acidity or basicity of the solid surface, while $A N^{*}$ and $D N$ the acceptor and donor numbers of polar probe. 


\section{RESULTS AND DISCUSSIONS}

\subsection{Surface energy of the cellulosic substrates}

As mentioned above, the dispersion component of surface tension, as well as the acid-base character of solid surface can be determined by IGC. The $\gamma_{\mathrm{s}}{ }^{\mathrm{d}}$ values of fibrous and ground cotton and linen samples were calculated from the retention data of n-alkanes by the Dorris-Gray method. As Fig. 1 and 2 show, the $\gamma_{s}{ }^{d}$ values decreased continuously with increasing the measurement temperature in the range of $30-70{ }^{\circ} \mathrm{C}$ independently of the type or form of samples. The slight decrease in the $\gamma_{\mathrm{s}}{ }^{\mathrm{d}}$ values as a function of increasing temperature, measured without any further changes in the fibre surface, can be explained by the thermal expansion of samples, as well as the decreasing adsorption ability of probes with increasing temperature [21].

\section{Figure 1.}

Figure 2.

Moreover, Fig. 1 clearly shows that dispersion component of surface tension of the fibrous cotton is a little bit higher than that of the linen fibre. These data are consistent with our earlier data [21] mentioned in the introduction section. Nevertheless, the ratio of $\gamma_{\mathrm{s}}^{\mathrm{d}}$ values of the two fibrous samples are rather surprising, since it was proved by XPS that surface of the linen fibre includes more groups containing oxygen than that of the cotton fibre [21]. Furthermore, as Fig. 2 shows, the $\gamma_{s}{ }^{d}$ of the ground cotton was smaller than $\gamma_{s}{ }^{d}$ of the ground linen at $70{ }^{\circ} \mathrm{C}$, as expected, but at lower temperatures similar tendency was observed as it was for the fibrous samples.

For comparison, surface energy of the two samples in fabric form was also tested by contact angle measurement using water and diiodomethane as wetting liquids. In Fig. 3 and 4 the drop images of water and diiodomethane, respectively, recorded immediately after reaching the fabric surface are presented. It is obvious that wettability of the linen fabric is better than that of the 
cotton, since the contact angles of diiodomethane and water drops are smaller on the surface of linen fabric (42 and $98^{\circ}$, respectively) than on the surface of cotton fabric (55 and $107^{\circ}$, respectively) (Table 2). It is well known, that the increasing surface energy usually results in higher wettability, so the results of contact angle measurements are in accordance with our assumption that the surface energy of linen is higher than that of cotton. In order to determine the surface tension of the cellulosic fibres, the Owens-Wendt formula (equation 12) [33] was applied and contact angles measured with diiodomethane and water were used for the calculation of dispersion and polar components, respectively.

$$
\gamma_{L V}(\cos \theta+1)=2\left(\gamma_{L V}^{d} \gamma_{S V}^{d}\right)^{1 / 2}+2\left(\gamma_{L V}^{p} \gamma_{S V}^{p}\right)^{1 / 2}
$$

where $\gamma_{L V}, \gamma_{L V}{ }^{d}$ and $\gamma_{L V} v^{p}$ are the surface tension of the liquid and its dispersion and polar components, respectively, used in the measurements. $\gamma_{s}{ }^{d}$ and $\gamma_{s}^{p}$ are the dispersion and polar components of surface tension of solid polymers (i.e. fabrics), respectively.

\section{Figure 3.}

Figure 4.

\section{Table 2}

Although, the high standard deviations of contact angles indicate the uncertainty of the determination, it is obvious that the surface energy of cotton is less than that of linen (Table 2). Comparing the surface tension values obtained from two different experiments (Fig. 1 and 2, Table 2), the question then arises: why so unrealistic high values were measured for cotton samples by IGC? Our hypothesis is that trapping of the n-alkane probes by the high molecular weight fatty acids, esters, alcohols and hydrocarbon chains of the outer waxy layer on the cotton surface can be the explanation of the unexpected results. In order to confirm the trapping theory, we 
ascertained the results from IGC by comparing them with those derived from the wetting experiments and by the knowledge available from earlier publications [34, 35].

As we demonstrated above, the $\gamma_{\mathrm{s}}{ }^{\mathrm{d}}$ values determined by IGC are larger than those calculated from the contact angles. This fact, however, is not unexpected if we consider that infinite dilution IGC measures exclusively the most active sites on the surface. Accepting the assumption of n-alkane trapping, it is understandable, that the surface energy for fibrous cotton is larger than that of fibrous linen at all temperatures. In spite of our expectation that the effect of trapping decreases with increasing temperature, Fig. 1 reveals that the difference between the $\gamma_{\mathrm{s}}{ }^{\mathrm{d}}$ values of the two substrates is almost independent of the measurement temperature.

Nevertheless, interpretation of the results shown in Fig. 2 is more difficult, since besides trapping, the effect of grinding has also to be considered. Previous work in this laboratory demonstrated that grinding increases the concentration of oxygen-containing groups on the surface and creates less perfect crystalline structure [21]. The former effect of grinding can result in an increase in the dispersion component of surface tension and also lead to a decrease in trapping of n-alkane probes especially on cotton. On the other hand, the less perfect crystal structure (i.e. the latter effect) which was more pronounced at linen, can bring about a less effective adsorption of probes on the substrates. That results in lower $\gamma_{\mathrm{s}}{ }^{\mathrm{d}}$ values measured by IGC especially on linen. The joint effect of these two actions can lead to the results presented in Fig. 2.

\subsection{Acid-base parameters of cellulosic substrates}

For obtaining the acid-base parameters of cotton and linen samples, the polar component of free energy $\left(\Delta G_{A}{ }^{a b}\right)$ of various polar probes' adsorption at different temperatures was measured. The most important observations for the first round of experiments were that while polar probes were not retained at all at any temperature on fibrous cotton, a mild retention was detected on the 
ground cotton, but exclusively in the temperature range of $30-50{ }^{\circ} \mathrm{C}$. For linen, however, the polar probes were retained both on the fibrous and ground samples at any temperature.

In order to obtain $\Delta G_{A}^{a b}$, first, a baseline with $n$-alkanes capable of only dispersion interaction was created. The approaches presented previously [28, 29] were used to define the baseline: the $R T \ln V_{N}$ values were plotted against the $a_{L V}\left(\gamma_{L V}{ }^{d}\right)^{1 / 2} \cdot \Delta G_{A}{ }^{a b}$ values of four polar probes, namely chloroform, ethyl acetate, tetrahydrofuran and acetone were determined. For chloroform and tetrahydrofuran, the $\Delta G_{A}^{a b}$ values are presented in Fig. 5a and b, respectively. It is obvious that the $\Delta G_{A}^{a b}$ values vary between 2.5 and $5.0 \mathrm{~kJ} / \mathrm{mol}$. Similar data were obtained with ethyl acetate (Fig. 6a), but a slightly larger $\Delta G_{A}{ }^{a b}$ values were measured with acetone (Fig. 6b).

\section{Figure 5.}

Figure 6.

Plotting the $\Delta G_{A}^{a b} / T$ values against the $1 / \mathrm{T}$ resulted in straight line, the slope of which equals $\Delta H_{A}{ }^{a b}$. The procedure followed and the straight line obtained is presented in Fig. 7 for chloroform adsorption on linen fibre. The derived $\Delta H_{A}{ }^{a b}$ values for all probes and adsorbents are summarized in Table 3 together with the regression coefficients of the lines, from which they were calculated. In spite of the difficulties described previously [28], results in Table 3 indicate that $\Delta H_{A}{ }^{a b}$ can be determined with acceptable accuracy. The regression coefficient of the straight lines exceeds 0.93 in each case, except for the acetone adsorption on cotton surface (0.7707). The similar values of $\Delta H_{A}^{a b}$ for chloroform and THF indicate that all the samples have amphoteric surface character. It is clear from Table 3, that the $\Delta H_{A}{ }^{a b}$ values for ethyl acetate and especially for acetone are higher than those calculated for the acidic and basic probes. The high specific enthalpies of 
amphoteric probes also confirm the amphoteric character of the surfaces investigated. The largest enthalpies can be measured on linen fibre, the smallest on ground linen.

\section{Figure 7}

\section{Table 3}

Understanding of these results is as difficult as it was in case of the dispersion component of surface tension. In our opinion the measured adsorption enthalpies are determined by the adsorption ability of polar probes on the given substrates, which depends on the surface energy and the crystalline structure of the surface. We think that the probability of trapping of polar probes on the waxy surface of cotton is smaller than that of n-alkanes, and the most important parameter is the less perfect crystalline surface created by grinding. As we proved earlier, the effect of grinding is stronger on linen than on cotton.

The acid-base parameters of cotton and linen samples were calculated from $\Delta H_{A}^{a b}$ using the $A N^{*}$ and $D N$ numbers proposed by Riddle, Fowkes and Guttmann $[31,32]$. According to Eq. 11 , the $\Delta H_{A}{ }^{a b} / A N^{*}$ was plotted against the $D N / A N^{*}$ in order to obtain the $K_{a}$ and $K_{d}$ parameters. The procedure is demonstrated in Fig. 8 for fibrous linen. The calculations, i.e. determination of $K_{a}$ and $K_{d}$ parameters were carried out for all samples and the results are listed in Table 4 together with the regression coefficients of the fit. It is obvious that the acid-base parameters can be calculated with practically the same accuracy for all samples. The calculated $K_{a}$ and $K_{d}$ parameters are very similar for cotton and linen samples. The larger donor numbers indicate basicity of the investigated cellulosic samples. The highest $K_{d} / K_{a}$ value of ground linen can correspond to high surface oxygen concentration of the substrate. Comparing these values with the results of contact angle measurements and with our former results of energy dispersive spectroscopy as well as Xray photoelectron spectroscopy [21], it can be concluded, that the measured parameters depend on 
the adsorbability of probe molecules on the investigated surface. Beside surface energy, grinding also influences the measured characteristics.

\section{Figure 8}

\section{Table 4}

It is worth noting, however, that the calculated $K_{d} / K_{a}$ values are not in accordance with the $\Delta H_{A}^{a b}$ values of different probes. While on the basis of $K_{d} / K_{a}$ ratios the investigated substrates show strong basic character, according to $\Delta H_{A}^{a b}$ values the surface character is evenly basic and acidic, namely amphoteric. The reason of the anomaly may be the incomparable scale of AN and DN numbers of polar probes used for determination [16].

\section{CONCLUSIONS}

Surface properties of fibrous and ground cotton and linen were investigated by IGC and contact angle measurement was applied for the fabrics of both fibres. In spite of the better wettability and higher surface tension of linen sample determined by contact angle measurement, significantly larger $\gamma_{s}{ }^{d}$ was measured for cotton than for linen by IGC. It is well-known that surface of cotton fibres is covered with a continuous waxy outer layer, which can acts as a trap for the nalkanes. Thus, trapping of n-alkanes among the molecules of cotton wax can be the reason of the unexpectedly high $\gamma_{s}{ }^{d}$ values determined for fibrous cotton by IGC. For ground samples, besides surface energy and trapping effects, the higher surface oxygen content and the less perfect crystalline surface can also influence the $\gamma_{s}{ }^{d}$ values.

Experiments with polar probes revealed that free energy of adsorption can be determined on all the linen samples, as well as on the ground cotton in the temperature range of $30-50{ }^{\circ} \mathrm{C}$, but polar probes were not retained at all on the fibrous cotton at any temperatures. For calculation of 
the acid-base character of surfaces, specific enthalpies were determined from the temperature dependence of free energies. Similar $\Delta H_{A}{ }^{a b}$ values were calculated with chloroform (acidic probe) and THF (basic probe), and relatively high $\Delta H_{A}{ }^{a b}$ values of the amphoteric ethyl acetate and acetone probes were measured for each of the investigated samples. These results indicated that the surface character of the cellulosic substrates is evenly basic and acidic, namely amphoteric.

Finally, it can be concluded that IGC is a suitable method for characterizing the surface properties of cellulosic substrates. If the influences of the different factors are considered, the determined properties can be understood and explained. With the aid of XPS, IGC gives valuable information on the surface physico-chemical properties of materials.

\section{ACKNOWLEDGEMENTS}

The financial support of the National Scientific Research Fund of Hungary (OTKA Grant No. K 108934) is highly appreciated.

\section{REFERENCES}

1. O. Faruk, A. K. Bledzki, H.-P. Fink, and M. Sain, Progr. Polym. Sci., 37, 1552 (2012).

2. E. Papirer, E. Brendle, H. Balard, and C. Vergelati, J. Adhes. Sci. Technol., 14, 321 (2000).

3. E. Cantergiani and D. Benczedi, J. Chromatogr. A, 969, 103 (2002).

4. P. Jandura, B. Riedl, and B. V. Kokta, J. Chromatogr. A, 969, 301 (2002).

5. G. Buschle-Diller, M. K. Inglesby, and Y. Wu, Colloids Surf., A, 260, 63 (2005).

6. B. A. P. Ass, M. N. Belgacem, and E. Frollini, Carbohydr. Polym., 63, 19 (2006).

7. X. Ren and G. Buschle-Diller, Colloid. Surface. A, 299, 15 (2007). 
8. N. Rjiba, M. Nardin, J.-Y. Drean, and R. Frydrych, J. Colloid Interface Sci., 314, 373 (2007).

9. J. Y. Y. Heng, D. F. Pearse, F. Thielmann, T. Lampke, and A. Bismarck, Compos. Interfaces, 14, 581 (2007).

10. N. Rjiba, M. Nardin, J.-Y. Drean, and R. Frydrych, J. Polym. Res., 17, 25 (2010).

11. L. Zhao and Y. Boluk, Appl. Surf. Sci., 257, 180 (2010).

12. N. Cordeiro, C. Gouveia, and M. J. John, Ind. Crops Prod., 33, 108 (2011).

13. N. Cordeiro, C. Gouveia, A. G. O. Moraes, and S. C. Amico, Carbohydr. Polym., 84, 110 (2011)

14. N. Cordeiro, C. Mendonca, L. A. Pothan, and A. Varma, Carbohydr. Polym., 88, 125 (2012)

15. E. S. Abdel-Halim, Carbohydr. Polym., 88, 1201 (2012).

16. J. A. F. Gamelas, Cellulose, 20, 2675 (2013).

17. S. Mohammadi-Jam and K. E. Waters, Adv. Colloid Interface Sci., 212, 21 (2014).

18. M. N. Belgacem, G. Czeremuszkin, S. Sapieha, and A. Gandini, Cellulose, 2, 145 (1995).

19. J. A. Trejo-O'Reilly, J. Y. Cavaille, N. M. Belgacem, and A. Gandini, J. Adhes., 67, 359 (1998).

20. E. Csiszar and E. Fekete, Text. Res. J., 80, 1307 (2010).

21. E. Csiszar and E. Fekete, Langmuir, 27, 8444 (2011).

22. E. Csiszar, E. Fekete, A. Toth, E. Bandi, B. Koczka, and I. Sajo, Carbohydr. Polym., 94, 927 (2013).

23. J. A. F. Gamelas, E. Ferraz, and F. Rocha, Colloids Surf., A, 455, 49 (2014).

24. J. R. Conder, Young, C.L., "Physicochemical Measurement by Gas Chromatography", Wiley, Chichester, 1979.

25. F. M. Fowkes, Ind. Eng. Chem., 56, 40 (1964).

26. J. Schultz, L. Lavielle, and C. Martin, J. Adhes., 23, 45 (1987). 
27. G. M. Dorris and D. G. Gray, J. Colloid Interface Sci., 77, 353 (1980).

28. E. Fekete, J. Moczo, and B. Pukansky, J. Colloid Interface Sci., 269, 143 (2004).

29. A. Voelkel, B. Strzemiecka, K. Adamska, and K. Milczewska, J. Chromatogr. A, 1216, 1551 (2009).

30. R. S. Drago, Wayland, B.B, J. Am. Chem. Soc., 87, 3571 (1965).

31. V. Gutmann, "The Donor-Acceptor Approach to Molecular Interactions", Plenum, New York, 1978.

32. F. L. Riddle and F. M. Fowkes, J. Am. Chem. Soc., 112, 3259 (1990).

33. D. K. Owens, Wendt, R.C., J. Appl. Polym. Sci, 13, 1741 (1969).

34. H. L. Lee and P. Luner, J. Colloid Interface Sci., 146, 195 (1991).

35. N. E. Zafeiropoulos, D. R. Williams, C. A. Baillie, and F. L. Matthews, Composites, Part A, 33, 1083 (2002). 
Table 1. Physico-chemical properties of the IGC probes used in the study [16, 31]

\begin{tabular}{|c|c|c|c|c|c|}
\hline Probe & $\begin{array}{c}a \\
(\AA)^{2}\end{array}$ & $\begin{array}{c}\gamma_{L}^{d} \\
\left(\mathrm{~mJ} \mathrm{~m}^{-2}\right)\end{array}$ & $\begin{array}{c}\mathrm{AN}^{*} \\
\left(\mathrm{~kJ} \mathrm{~mol}^{-1}\right)\end{array}$ & $\begin{array}{c}\mathrm{DN} \\
\left(\mathrm{kJ} \mathrm{mol}^{-1}\right)\end{array}$ & Specific character \\
\hline$n$-Hexane & 51.5 & 18.4 & - & - & Neutral \\
\hline$n$-Heptane & 57.0 & 20.3 & - & - & Neutral \\
\hline n-Octane & 62.8 & 21.3 & - & - & Neutral \\
\hline$n$-Nonane & 69.0 & 22.7 & - & - & Neutral \\
\hline n-Decane & 74.0 & 24.2 & - & - & Neutral \\
\hline Chloroform & 44.0 & 25.0 & 22.6 & 0 & Acid \\
\hline Ethyl acetate & 48.0 & 19.6 & 6.3 & 71.6 & Amphoteric \\
\hline Acetone & 42.5 & 16.5 & 10.5 & 71.2 & Amphoteric \\
\hline Tetrahydrofuran & 45.0 & 22.5 & 2.1 & 83.8 & Base \\
\hline
\end{tabular}


Table 2. Results of contact angle measurements for linen and cotton fabrics

\begin{tabular}{|c|c|c|}
\hline & Linen & Cotton \\
\hline$\theta_{\text {diidomethane }}\left({ }^{\circ}\right)$ & $42 \pm 4$ & $55 \pm 4$ \\
\hline$\left.\theta_{\text {water }}{ }^{\circ}\right)$ & $98 \pm 5$ & $107 \pm 4$ \\
\hline$\gamma_{\mathrm{s}}{ }^{\mathrm{d}}\left(\mathrm{mJ} / \mathrm{m}^{2}\right)$ & 38.6 & 31.4 \\
\hline$\gamma_{\mathrm{s}}^{\mathrm{p}}\left(\mathrm{mJ} / \mathrm{m}^{2}\right)$ & 0.12 & 0 \\
\hline
\end{tabular}


Table 3. Acid-base component of the adsorption enthalpy of polar probes for fibrous and ground linen and for ground cotton

\begin{tabular}{|c|c|c|c|c|c|c|}
\hline \multirow{2}{*}{} & \multicolumn{2}{|c|}{ Linen, fibrous } & \multicolumn{2}{c|}{ Linen, ground } & \multicolumn{2}{c|}{ Cotton, ground } \\
\cline { 2 - 7 } & $\Delta H_{A}{ }^{a b}$ & \multirow{2}{*}{$R^{2}$} & $\Delta H_{A}{ }^{a b}$ & $R^{2}$ & $\Delta H_{A}{ }^{a b}$ & $R^{2}$ \\
& $(\mathrm{~kJ} / \mathrm{mol})$ & & $(\mathrm{kJ} / \mathrm{mol})$ & & $(\mathrm{kJ} / \mathrm{mol})$ & \\
\hline Chloroform & 19.3 & 0.9942 & 11.2 & 0.9682 & 18.8 & 0.9956 \\
\hline Ethyl acetate & 22.5 & 0.9907 & 17.5 & 0.9333 & 19.0 & 0.9523 \\
\hline Tetrahydrofuran & 17.3 & 0.9872 & 11.5 & 0.9377 & 15.3 & 0.9998 \\
\hline Acetone & 26.2 & 0.9800 & 21.1 & 0.9940 & 24.1 & 0.7701 \\
\hline
\end{tabular}


Table 4. Acid-base properties of fibrous and ground linen and ground cotton, and the standard deviation of determination

\begin{tabular}{|c|c|c|c|c|}
\hline Samples & $K_{a}$ & $K_{d}$ & $K_{d} / K_{a}$ & $\mathrm{R}^{2}$ \\
\hline Linen, fibrous & 0.18 & 1.18 & 6.55 & 0.9918 \\
\hline Linen, ground & 0.12 & 1.00 & 8.33 & 0.9587 \\
\hline Cotton, ground & 0.16 & 1.07 & 6.68 & 0.9952 \\
\hline
\end{tabular}




\section{Figure captions}

Fig. 1 Effect of temperature on the dispersion component of surface tension $\left(\gamma_{\mathrm{s}}{ }^{\mathrm{d}}\right)$ of fibrous cotton and linen samples

Fig. 2 Effect of temperature on the dispersion component of surface tension $\left(\gamma_{\mathrm{s}}{ }^{\mathrm{d}}\right)$ of ground cotton and linen samples

Fig. 3 Water contact angles on the surface of raw cotton (a) and linen (b) fabrics

Fig. 4 Diiodomethane contact angles on the surface of raw cotton (a) and linen (b) fabrics

Fig. $5 \Delta G_{A}{ }^{a b}$ of chloroform (a) and THF (b) adsorption at different temperatures for fibrous and ground linen and for ground cotton

Fig. $6 \Delta G_{A}^{a b}$ of adsorption of amphoteric probes (a: ethyl acetate, b: acetone) at different temperatures for fibrous and ground linen and for ground cotton

Fig. 7 Determination of specific enthalpy of adsorption of chloroform on linen fibre

Fig. 8 Determination of acid-base constants of linen fibre 
Fekete, Fig.1

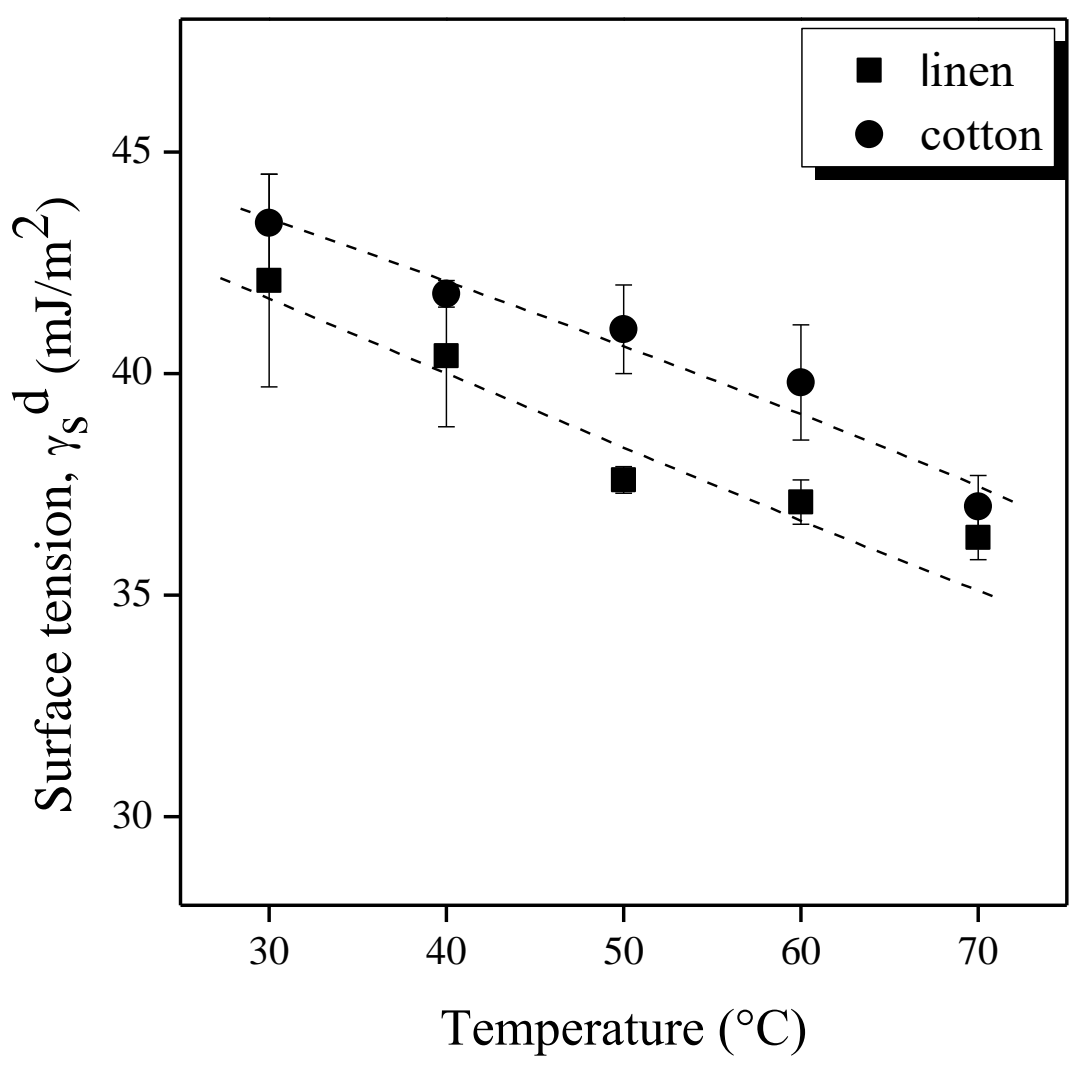

Fig. 1 Effect of temperature on the dispersion component of surface tension $\left(\gamma_{\mathrm{s}}{ }^{\mathrm{d}}\right)$ of fibrous cotton and linen samples 
Fekete, Fig.2

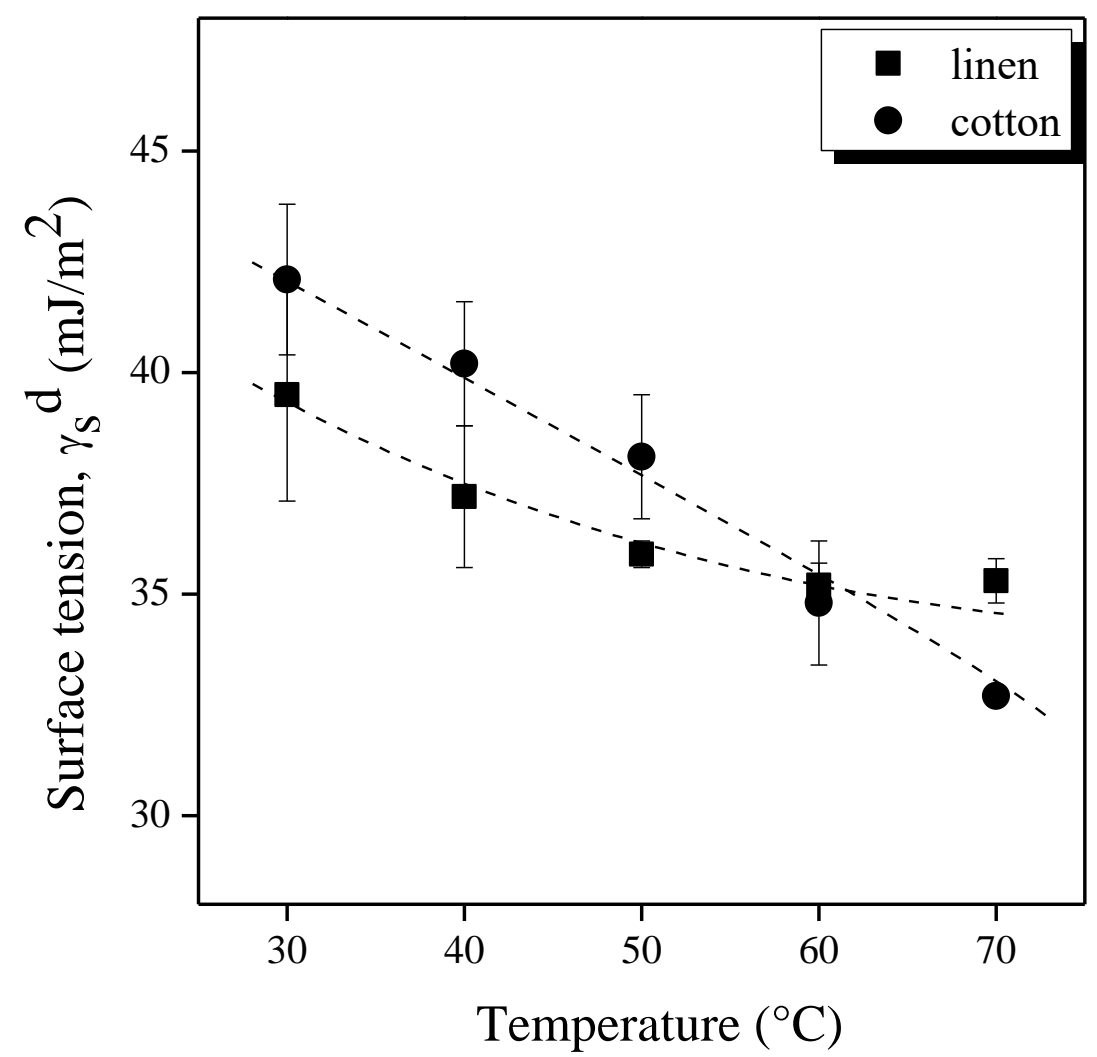

Fig. 2 Effect of temperature on the dispersion component of surface tension $\left(\gamma_{\mathrm{s}}{ }^{\mathrm{d}}\right)$ of ground cotton and linen samples 
Fekete, Fig. 3

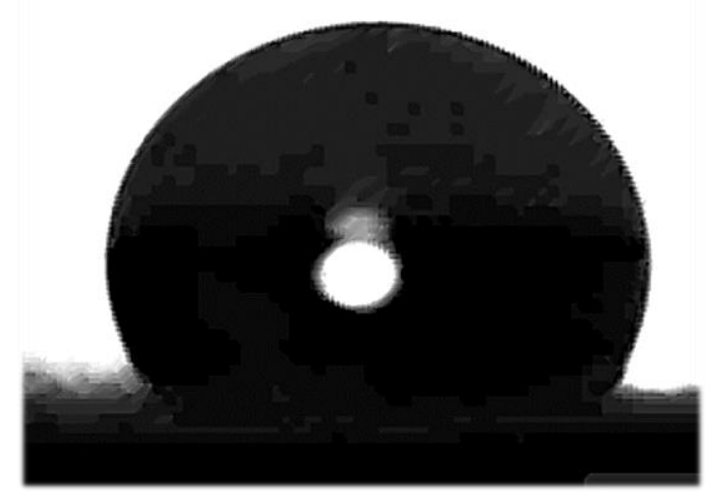

a)

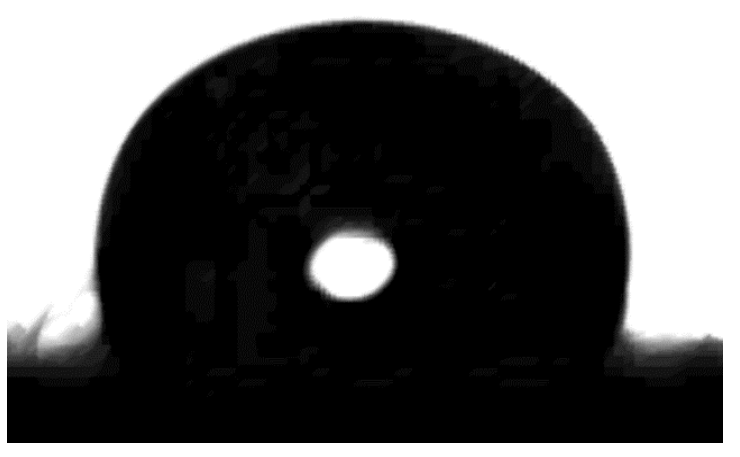

b)

Fig. 3 Water contact angles on the surface of raw cotton (a) and linen (b) fabrics 
Fekete, Fig.4

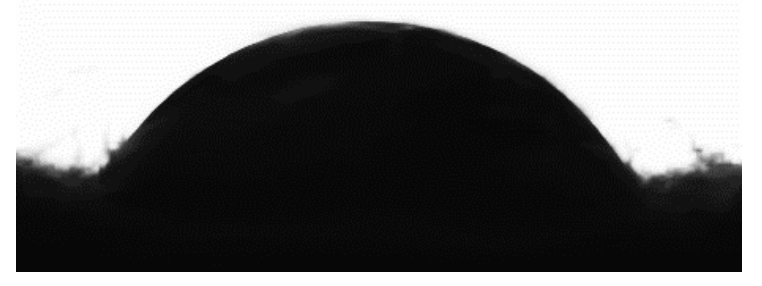

a)

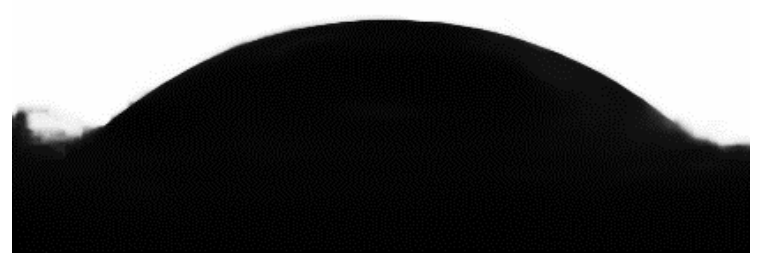

b)

Fig. 4 Diiodomethane contact angles on the surface of raw cotton (a) and linen (b) fabrics 
Fekete, Fig.5

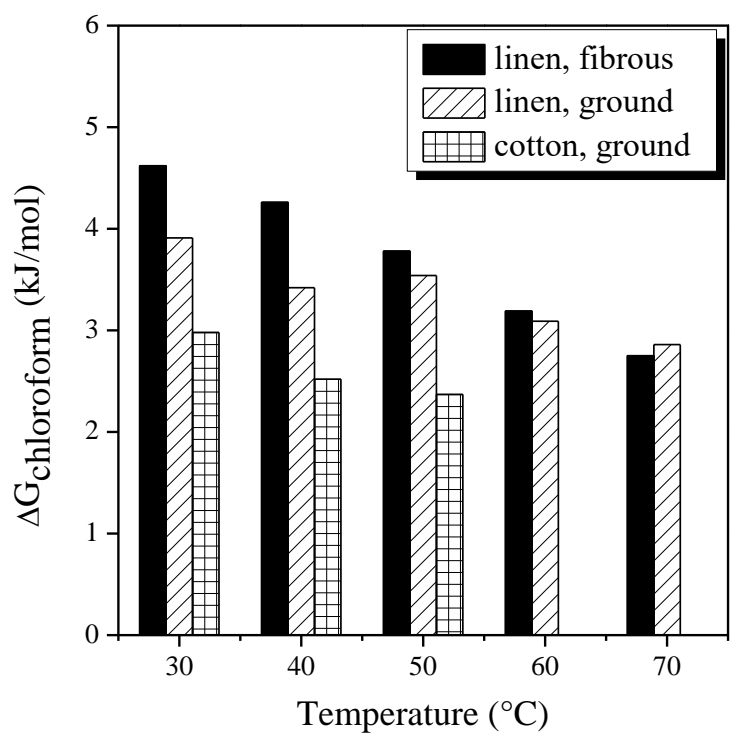

a)

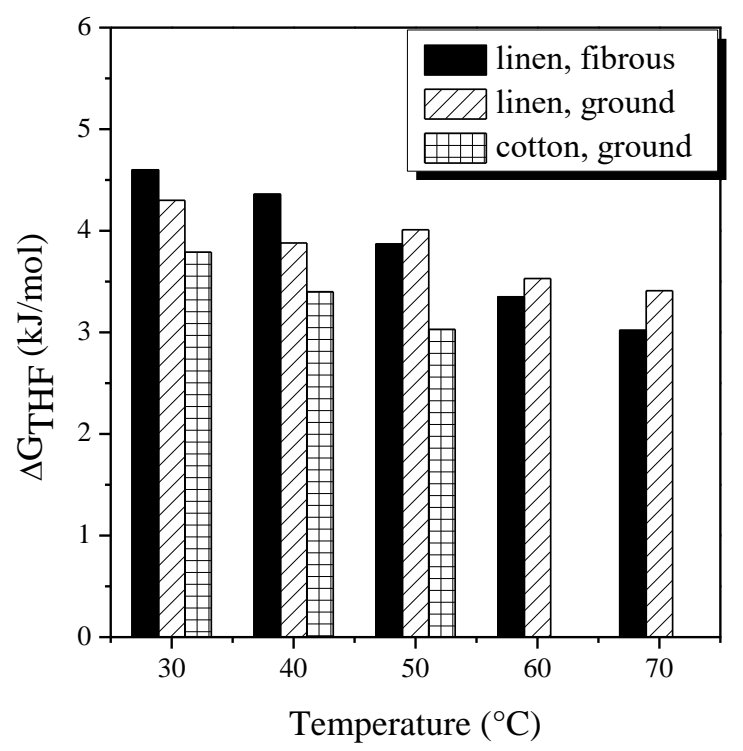

b)

Fig. $5 \Delta G_{A}{ }^{a b}$ of chloroform (a) and THF (b) adsorption at different temperatures for fibrous and ground linen and for ground cotton 
Fekete, Fig.6

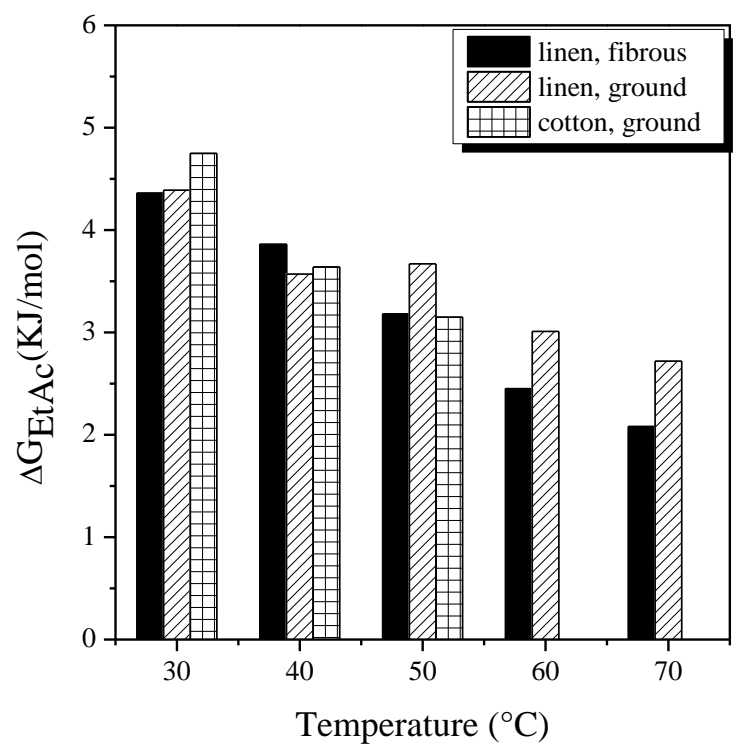

a)

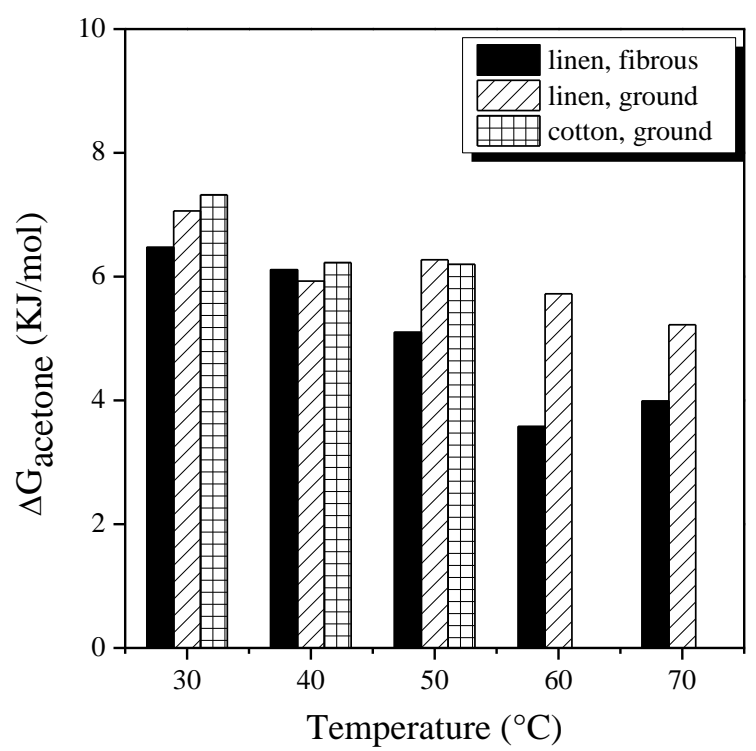

b)

Fig. $6 \Delta G_{A}^{a b}$ of adsorption of amphoteric probes (a: ethyl acetate, b: acetone) at different temperatures for fibrous and ground linen and for ground cotton 
Fekete, Fig. 7

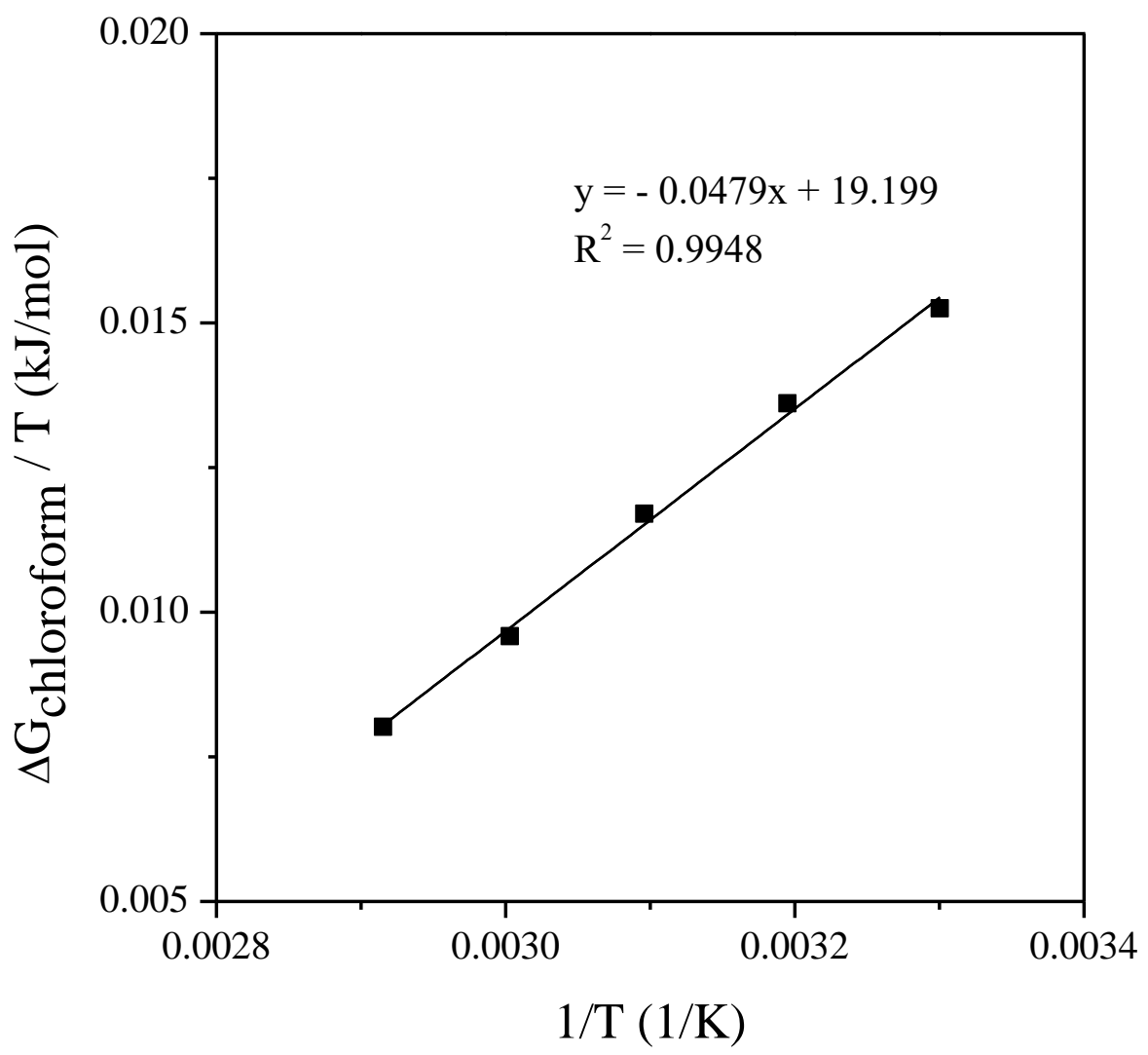

Fig. 7 Determination of specific enthalpy of adsorption of chloroform on linen fibre 
Fekete, Fig.8

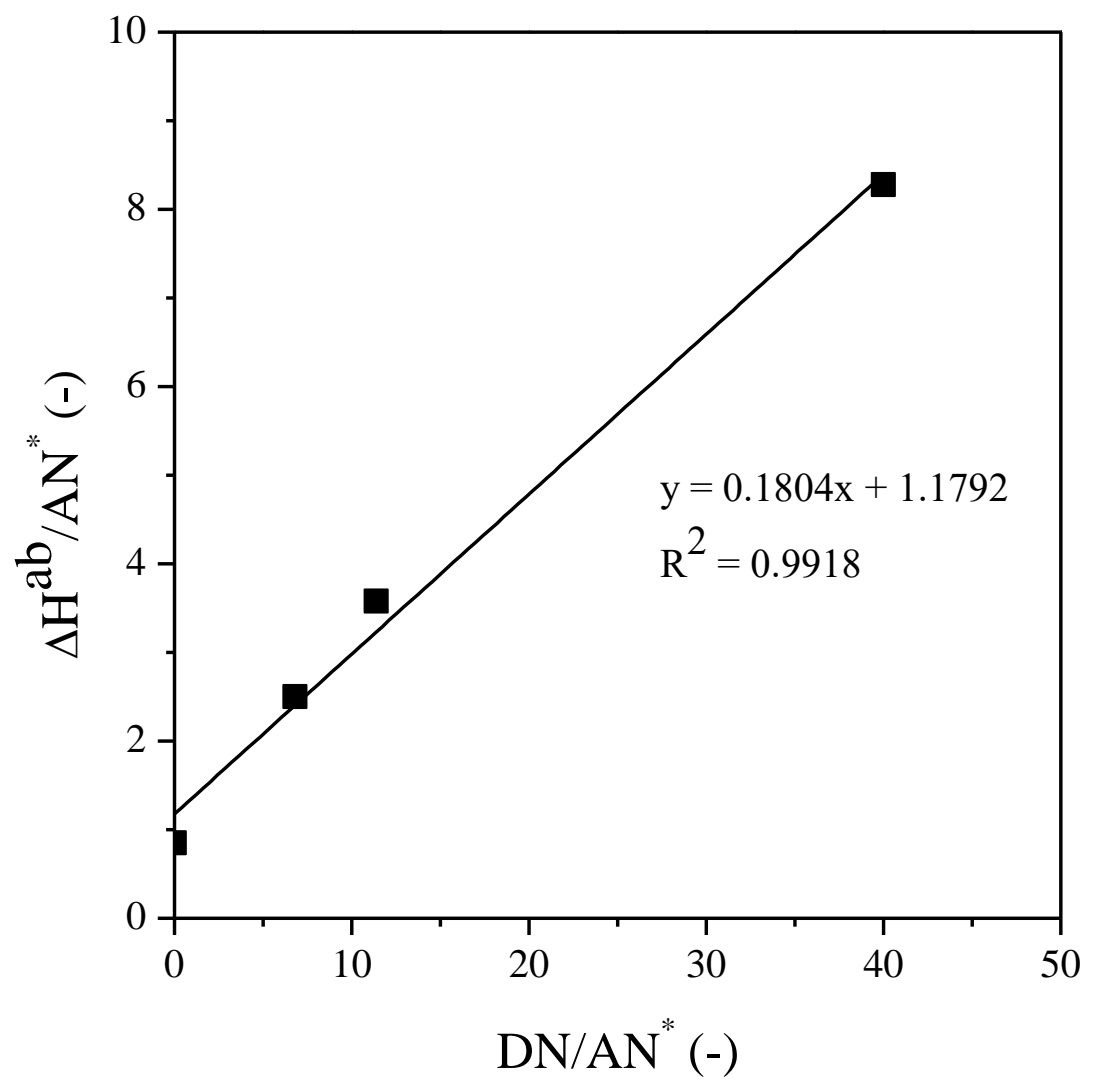

Fig. 8 Determination of acid-base constants of linen fibre 